\title{
Antireflux mucosectomy (ARMS) and antireflux mucosal ablation (ARMA) for gastroesophageal reflux disease: a systematic review and meta-analysis
}

\section{(ㄷ)(우우}

Authors

Enrique Rodríguez de Santiago*, 1, Carlos Teruel Sanchez-Vegazo*, 1, Beatriz Peñas ${ }^{1}$, Yuto Shimamura², Mayo Tanabe², Noelia Álvarez-Díaz ${ }^{3}$, Sofía Parejo ${ }^{1}$, Sumi Kazuya², Natalia Marcos-Carrasco ${ }^{1}$, Enrique Vazquez-Sequeiros ${ }^{1}$, Haruhiro Inoue $^{2}$, Agustín Albillos ${ }^{1}$

Institutions

1 Department of Gastroenterology and Hepatology, Hospital Universitario Ramón y Cajal, Instituto Ramón y Cajal de Investigación Sanitaria (IRYCIS), CIBEREHD, Universidad de Alcalá, Madrid, Spain

2 Digestive Disease Center, Showa University Koto Toyosu Hospital, Tokyo, Japan

3 Medical library, Hospital Universitario Ramón y Cajal, Madrid, Spain

submitted 17.4.2021

accepted after revision 9.7.2021

\section{Bibliography}

Endosc Int Open 2021; 09: E1740-E1751

DOI 10.1055/a-1552-3239

ISSN 2364-3722

(C) 2021. The Author(s).

This is an open access article published by Thieme under the terms of the Creative Commons Attribution-NonDerivative-NonCommercial License, permitting copying and reproduction so long as the original work is given appropriate credit. Contents may not be used for commercial purposes, or adapted, remixed, transformed or built upon. (https://creativecommons.org/licenses/by-nc-nd/4.0/)

Georg Thieme Verlag KG, Rüdigerstraße 14,

70469 Stuttgart, Germany

Corresponding author Enrique Rodríguez de Santiago, Endoscopy Unit, Hospital Universitario Ramón y Cajal, M-607, km. 9, 100, 28034 Madrid, Spain

Fax: +1234567890

enrodesan@gmail.com

\# Supplementary material is available under https://doi.org/10.1055/a-1552-3239

\section{ABSTRACT}

Background and study aims Antireflux mucosectomy (ARMS) and antireflux mucosal ablation (ARMA) are new endoscopic procedures for patients with gastroesophageal reflux disease (GERD). We conducted a meta-analysis to systematically assess the feasibility, clinical success, and safety of these procedures.

Patients and methods We searched Embase, PubMed, and Cochrane Central from inception to October 2020. Overlapping reports, animal studies, and case reports were excluded. Our primary outcomes were clinical success and adverse events (AEs). Secondary outcomes included technical success, endoscopic esophagitis, 24-hour pH monitoring, and proton pump inhibitor (PPI) use. A random effects model was used to pool data.

Results In total, 15 nonrandomized studies (12 ARMS, $\mathrm{n}=$ 331; 3 ARMA, $n=130$ ) were included; 10 were conducted in patients with refractory GERD. The technical success rate was $100 \%$. The pooled short-term (first assessment within the first 6 months), 1-year, and 3-year clinical success rates were $78 \%$ (95\% confidence interval [95\% Cl] 70\%-85\%), $72 \%(95 \% \mathrm{Cl} 47 \%-92 \%)$, and $73 \%(95 \% \mathrm{Cl} 65 \%-81 \%)$, respectively. ARMS and ARMA yielded similar clinical success. The proportion of patients off PPIs at 1 year was $64 \%$ (95\% Cl 52\%-75\%). There were significant drops $(P<0.01)$ in validated clinical questionnaires scores, presence of esophagitis, and acid exposure time. The most common AE $(11 \%, 95 \% \mathrm{Cl} 8 \%-15 \%)$ was dysphagia requiring dilation (7\%, $95 \% \mathrm{Cl} 5 \%-11 \%)$. Four cases of perforation were recorded, all in patients undergoing ARMS.

Conclusions Our meta-analysis of nonrandomized studies suggests that ARMS and ARMA are safe and effective for patients with GERD.

\footnotetext{
* These authors contributed equally.
} 


\section{Introduction}

Gastroesophageal reflux disease (GERD) is a common disorder with an estimated worldwide prevalence of $14.8 \%$ [1]. Despite being regarded as a benign condition, GERD is the main risk factor for Barrett's esophagus, can significantly affect patient quality of life, and carries a steadily increasing cost for health care systems [2]. Proton pump inhibitors (PPIs) are the mainstay of therapy, but about $10 \%$ to $40 \%$ of patients do not achieve a satisfactory response [3]. In addition, many of the remaining patients require a daily dose to stay in remission, and long-term PPI therapy has been associated with several adverse effects, such as higher risks of pneumonia and dementia [4]. Laparoscopic antireflux surgery (LARS) represents the main nonpharmacological alternative to PPIs and is supported by current clinical guidelines [3]. Nonetheless, this procedure poses several problems, including periprocedural morbidity, dysphagia, gas-bloat syndrome, and a considerable need for reintervention [5]. This has prompted the development of less invasive endoscopic procedures, such as transoral incisionless fundoplication, injection of sclerosants into the gastroesophageal junction (GOJ), and the Stretta procedure [6]. However, these techniques have not spread worldwide due to the need for add-on devices, limited long-term data from randomized clinical trials (RCTs) and decreased effectiveness over time [6].

In 2014, Inoue et al. [7] reported the first case series of patients treated with antireflux mucosectomy (ARMS) with favorable outcomes. ARMS, which involves resection of the gastric cardiac mucosa, reduces the opening of the $\mathrm{GO}$ J through the healing process of the resulting scar. Based on the same principle, ablation of the mucosa by coagulation current or argon plasma coagulation (APC) has also recently been suggested to induce scar formation and yield similar results $[8,9]$. This approach, called antireflux mucosal ablation (ARMA), would hypothetically simplify the procedure, reduce the risk of perforation, and ease the retreatment of patients who have failed ARMS.Subsequent reports of both techniques from other groups are encouraging [9-12] but scarce and difficult to translate into clinical practice. A meta-analysis of existing studies may help us to obtain more robust data to support or refute the use of this new technique.

Herein, we systematically gathered and appraised the available evidence on ARMS and ARMA. Our primary aims were to assess clinical success and safety. We also examined feasibility, changes in PPI requirements, and the impact of these procedures on complementary GERD diagnostic tests.

\section{Patients and methods}

\section{Study registration and design}

The protocol was registered at the International Prospective Register of Systematic Reviews before the literature search (registration number: CRD42020214688). The study was carried out according to Preferred Reporting Items for Systematic Reviews and Meta-Analyses (PRISMA) guidelines. Risk of bias was assessed by the Quality Assessment Tool for Before-After (PrePost) Studies With No Control Group from the National Insti- tutes of Health. This tool includes 12 items and is considered appropriate for uncontrolled before-after studies [13]. Two authors (ERDS and CT) independently screened all titles and abstracts, reviewed full texts, extracted data, and assessed risk of bias using pre-designed electronic forms. A third author (BP) checked the extracted data for accuracy and assessed the risk of bias. Disagreements were solved by consensus among these three authors.

\section{Selection criteria and search strategy}

We included all studies reporting on ARMS and ARMA regardless of the type of endoscopic method used (i.e., endoscopic mucosal resection [EMR], endoscopic submucosal dissection [ESD], APC, or ablation by coagulation current). The exclusion criteria were as follows: animal studies, case reports, concomitant endoscopic suturing or plication, and duplicate studies. Abstract conferences were included, as recommended by the Cochrane handbook [14]. In the case of overlapping data from the same institution, we selected the study included by type of report (full-text publication over conference abstract) and study date (the most recent).

The search strategy was designed by an expert librarian (NA) and is detailed in Supplementary Material. MEDLINE, Embase, and the Cochrane Central Register of Controlled Trials were searched from database inception to October 2020. No language restriction was applied. Secondarily, the reference lists of the included articles and the abstract books of the leading endoscopy scientific societies were handsearched for additional studies (see Supplementary Material). We also checked citations from Google Scholar through to November 2020.

\section{Study outcomes and data collection}

Our primary outcomes were clinical success and adverse events. Clinical success was measured using the definition provided by the authors. When a definition was not reported, we used the number of patients with complete postprocedural cessation of PPIs. When available, we also measured clinical success by the improvement in the score of validated clinical questionnaires: GERD health-related quality of life (GERDHRQL) and GerdQ. Secondary outcomes included technical success (defined as completion of the intended procedure), PPI consumption, esophagitis at endoscopy, 24-hour pH monitoring, and high-resolution manometry (HRM) parameters. We calculated short-term outcomes by using data from the first assessment within the first 6 months after ARMS or ARMA. Oneyear and 3-year outcomes were also recorded.

We recorded the following items from each study: authors and date, design, location, type of endoscopic intervention, sample size, selection criteria, study definitions, age and sex of participants, technical and clinical success, adverse events (AEs) (i.e., clinically significant gastrointestinal bleeding [defined as bleeding that prompted blood transfusion, reintervention, admission, or prolonged hospital stay], perforation, pneumonia, infection, dysphagia requiring dilation, and procedurerelated mortality), and pre- and postprocedural GERD-related outcomes (GERD symptoms, PPI use, acid exposure time, DeMeester score, esophagitis at endoscopy, number of reflux epi- 
sodes, integrated relaxation pressure, and resting lower esophageal sphincter pressure). We contacted principal authors when deemed appropriate in the case of incomplete data. We also planned to collect 24-hour $\mathrm{pH}$ impedance data, but no detailed data were found.

\section{Statistical analysis}

Our primary analysis was based on the success of the first ARMS or ARMA. Patients who required a second procedure were treated as failures. We pooled ARMS and ARMA outcomes in our primary analysis because both procedures are technically similar and share the same mechanism of action (cardiac scarring). We used a random effects model for our analysis, given the clinical context. This decision was made beforehand and not based on post-hoc heterogeneity. The Freeman-Tukey double arcsine transformation was used to pool binary outcomes [15]. Preand postquantitative variables were meta-analyzed by using the inverse-variance weighting unstandardized mean difference (MD). Means and standard deviations were imputed when required according to the formulae described by Wan et al. [16]. Heterogeneity was assessed by visual inspection of forest plots and the $\mathrm{I}^{2}$ statistic. $\mathrm{I}^{2}$ values were interpreted according to the Cochrane handbook: $0 \%$ to $40 \%=$ might not be important; $30 \%$ to $60 \%=$ may represent moderate heterogeneity; $50 \%$ to $90 \%=$ may represent substantial heterogeneity; and $75 \%$ to $100 \%=$ considerable heterogeneity [14]. Publication bias was assessed by funnel plots. In addition, the Egger's regression test was conducted when the number of studies exceeded 10. Statistical analyses were conducted with Stata 14.1 (Stata Corp., Texas, United States). Significance was set at $P<$ 0.05 .

\section{Subgroups and sensitivity analyses}

Given the number of available studies, we used subgroup analysis rather than metaregression to assess heterogeneity and explore the robustness of our findings. We performed the following analyses: i) ARMS vs. ARMA; ii) mucosal esophageal resection or ablation vs. a gastric cardiac-only procedure; iii) clinical success defined as a $\geq 50 \%$ drop in a validated clinical questionnaire, because this definition has been used in several GERD trials [17]; iv) good/fair- vs. low-quality studies; and v) conference abstracts vs. full-text publications.

\section{Results}

Our initial search yielded 2,149 citations, and 1,554 titles and abstracts were reviewed after duplicate removal. A total of 15 studies ( $n=461)$ were finally included, as detailed in the PRISMA flowchart (> Fig. 1).

\section{Characteristics of the studies, study population, and risk of bias}

In total, 331 patients from 12 studies underwent 335 ARMS procedures [10-12,18-26], whereas 130 patients from three studies underwent 150 ARMA procedures $[8,9,27]$. Twenty-six patients underwent a second procedure: 4 underwent a second ARMS [10,12], 20 underwent a second ARMA [9], and 2 who

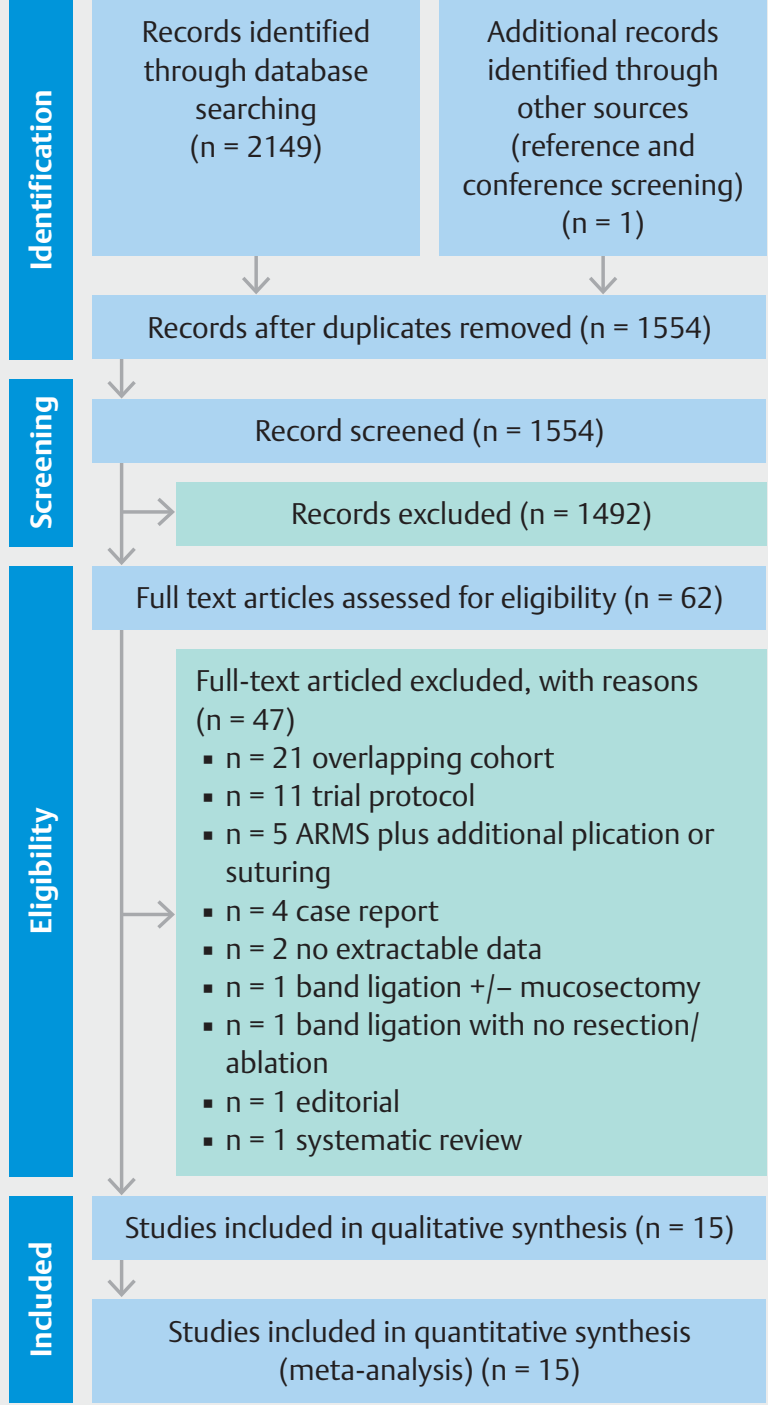

Fig. 1 PRISMA flowchart.

failed ARMS were treated with ARMA [8]. Eight studies were conducted in Asia $[8,10,11,18,19,21,24,26,27]$, four in Europe $[12,20,23,25]$, and three in North America [9,22, 27]. Nine studies were reported as full-text publications $[8-10,12$, 18-22] and six as conference abstracts [11,23-27]. Fourteen studies were single-arm, and one study compared the outcomes of ARMS with those of patients who underwent LARS [22].

Ten studies included patients with refractory GERD unresponsive to PPI therapy [8-12, 18, 20, 21, 24, 26], two included PPI-dependent patients $[19,25]$, and the remaining three studies did not detail the response to PPIs [22, 23, 27]. Sliding hiatal hernia $\geq 2$ to $3 \mathrm{~cm}$ was an exclusion criterion in all studies. Two ARMS studies included patients with prior LARS or bariatric surgery $[12,20]$, and one case series tested ARMA in patients with GERD after peroral endoscopic myotomy [27]. The remaining studies comprised patients without a history of any endoscopic 
or surgical therapy in the GOJ. The mean/median procedure time was under 70 minutes in all studies with available data $[8-10,12,18,20-22,27])$. The intervention was performed under deep sedation in three studies $[9,10,27]$ and under general anesthesia in four $[12,19,20,22]$; both methods were used in two $[8,18]$ (six studies had no available data). The procedure was carried out on an outpatient basis in five studies [9, 12, 20, 20,22 ], patients were admitted in one study [19], and the decision depended on patients' characteristics in two $[8,18]$ (seven studies had no available data). Three studies reported clinical success at 1 year $[9,18,19]$ and two at 3 years $[9,18]$. Additional details are provided in > Table 1 and Supplementary Table 1.

Seven studies were considered to be of good or fair quality and 8 of poor quality, as detailed in $>$ Table 2 . Only five studies clearly specified that all eligible patients that fulfilled the entry criteria were actually treated with ARMS or ARMA during the study period $[7,19,23,24,26]$. The major methodological drawbacks were a lack of blinded assessment and a low sample size. Funnel plots and Egger's regression test results suggested that studies with a small sample size reported fewer adverse events. No publication bias was detected for other outcomes (Supplementary Fig. 1).

\section{Technical and clinical success}

Technical success was achieved in all cases $(100 \%, 95 \%$ confidence interval $[\mathrm{Cl}] 100 \%-100 \%$ ). The pooled short-term (first assessment within the first 6 months), 1-year, and 3-year clinical success rates were $78 \%(95 \% \mathrm{Cl} 70 \%-85 \%)$, and $72 \%(95 \% \mathrm{Cl}$ $47 \%-92 \%$ ), respectively ( $\triangleright$ Table 3 and $>$ Fig. 2 ). There was a significant drop in GERD-HRQL and GerdQ scores, as shown in - Table 3. The pooled proportions of patients off PPIs at shortterm follow-up and at 1 year were $63 \%$ and $64 \%$, respectively ( $\triangleright$ Table 3). See Supplementary material for 3-year data.

A total of 47 patients underwent a rescue procedure: 22 patients received a second ARMA, 4 a second ARMS, and 21 were treated by LARS ( 14 fundoplications, 6 magnetic sphincter augmentations, and 1 gastric by-pass $[8,9,10,12,19,22])$. Shortterm data for a second ARMS/ARMA were available for 16 of the 26 patients, 12 of whom achieved clinical success $(80 \%$, $\left.95 \% \mathrm{Cl} 51 \%-99 \%, \mathrm{I}^{2} 0 \%\right)$. LARS was feasible in all patients, but postprocedural GERD outcomes for patients were not reported.

\section{Adverse events}

The overall rate of AEs was $11 \%$ ( 15 studies, $n=451,95 \% \mathrm{Cl} 8 \%-$ $15 \%, I^{2} 0 \%$ ) ( $\triangleright$ Table 1 and $>$ Fig. 2 ). The most common endoscopy-related $A E$ was dysphagia requiring dilation $(7 \%, 95 \% \mathrm{Cl}$ $5 \%-11 \%, I^{2} 0 \%$ ) ( Fig. 2). All patients were successfully treated by endoscopic balloon dilation (range, 12-15 mm) (Supplementary Table 2). Clinically significant gastrointestinal bleeding was reported in nine patients $\left(2 \%, 95 \% \mathrm{Cl} 1 \%-4 \%, \mathrm{I}^{2} 0 \%\right)$. Perforation occurred in four patients $\left(1 \%, 95 \% \mathrm{Cl} 0 \%-2 \%, \mathrm{I}^{2}\right.$ $0 \%)$, two of whom required surgery [19]. A detailed description of all AEs is provided in Supplementary Table 3. No deaths were recorded.

Regarding repeat procedures, no AEs were reported in the 22 patients that underwent ARMA after previous ARMA or ARMS $[8,9]$. Yoo et al. reported that two patients who under- went redo ARMS did not experience any AEs [10], while Monino et al. did not detail if the two patients in their series that underwent a second ARMS suffered any AEs [12].

\section{Endoscopic, 24-hour pH, and high-resolution manometry monitoring}

There was a significant short-term change in acid exposure time (six studies, $\mathrm{n}=188, \mathrm{MD}=12 \%, P=0.01,95 \% \mathrm{Cl} 2.6-21.4$, $I^{2} 94 \%$; Mean pre-procedure $17.1 \%$, mean post-procedure 5.1\%) and DeMeester score (six studies, $\mathrm{n}=244, \mathrm{MD}=40.1, P<$ 0.01, 95\% Cl 13.5-66.6, I2 $98 \%$; Mean pre-procedure 56.0, mean post-procedure 15.9) (Supplementary Table 2). The pooled proportions of patients with normal acid exposure time $(<4.2 \%)$ and DeMeester score $(<14.7)$ after the procedure were $71 \%$ (three studies, $\mathrm{n}=143 ; 95 \% \mathrm{Cl} 38 \%-96 \%, 1289 \%$ ) and $63 \%$ (three studies, $\mathrm{n}=97 ; 95 \% \mathrm{Cl} 44 \%-80 \%, 1258 \%$ ), respectively. We did not meta-analyze 24-hour $\mathrm{pH}$ monitoring at 1 year because only one ARMA study reported this variable [9]. That study found a significant drop in both parameters [9].

The pooled proportion of esophagitis at endoscopy significantly dropped $(P<0.01)$ from $86 \%$ at baseline $(95 \% \mathrm{Cl} 60 \%-$ $\left.100 \%, I^{2} 90.3 \%\right)$ to $14 \%\left(95 \% \mathrm{Cl} 5 \%-27 \%, \mathrm{I}^{2} 63.9 \%\right)$ at the short-term evaluation (four studies, $n=189$ ) (Supplementary Table 2). The proportion of esophagitis at 1-year was not pooled because only one ARMA study reported the esophagitis rate: $9 \%$ at 1 year, as well as $4.8 \%$ at 3 years [9].

Only one ARMS study reported the changes in HRM at 6 months. This study found a significant increase in the lower esophageal sphincter pressure (from a mean of 16.7 to $20.7 \mathrm{~mm} \mathrm{Hg}, P<0.01)$ and the integrated relaxation pressure (from a mean of 7.3 to $9.4 \mathrm{~mm} \mathrm{Hg}, P=0.05$ ). In addition, GOJ distensibility, as measured by the EndoFLIP distensibility index, also fell from 19.0 to $13.9(P<0.01)$ [10].

\section{Subgroup and sensitivity analysis}

We compared ARMS and ARMA outcomes via subgroup analysis. The short-term clinical success of ARMA $(84 \%, 95 \% \mathrm{Cl} 67 \%-$ $97 \%$ ) was slightly higher than that of ARMS (76\%, 95\%Cl 69\%$82 \%$ ), although the difference was not significant. This subgroup analysis reduced the heterogeneity found in short-term clinical success from moderate to low. The proportion of patients off PPIs and AEs were similar ( $\triangleright$ Table 4$)$. The four cases of perforation occurred in patients treated with ARMS ( $P=$ 0.12 ).

Esophageal mucosal resection did not influence clinical success or the proportion of AEs (Supplementary Table 4). The pooled proportion of patients that achieved $a \geq 50 \%$ drop in validated clinical questionnaire scores was $74 \%$ (six studies, $\mathrm{n}=$ $234,95 \% \mathrm{Cl} 64 \%-82 \%, \mathrm{I}^{2} 46.9 \%$ ) (Supplementary Fig. 2). The findings of our primary analysis were not influenced by the type of publication or by study quality (Supplementary Tables 5 and 6). 


\begin{tabular}{|c|c|c|c|c|c|}
\hline 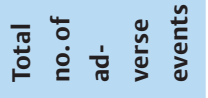 & $\sim$ & $\stackrel{\circ}{\circ}$ & in & $N$ & $\stackrel{\bullet}{\leftarrow}$ \\
\hline 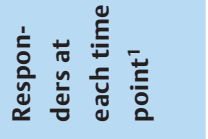 & $\frac{6}{\ln }$ & $\frac{\tilde{D}}{\frac{\sigma}{\gamma}}$ & $\frac{m}{o}$ & 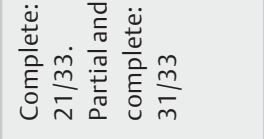 & $\frac{\infty}{\bar{\sigma}} \quad \frac{\bar{v}}{\underline{\sigma}}$ \\
\hline 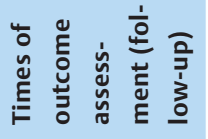 & 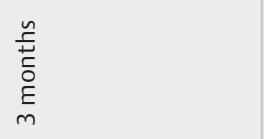 & 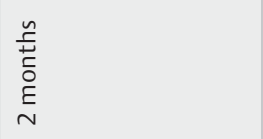 & 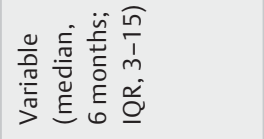 & 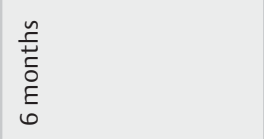 & 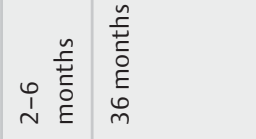 \\
\hline$z$ & 6 & $\widetilde{6}$ & $\stackrel{m}{m}$ & $\stackrel{m}{m}$ & $\stackrel{\text { I }}{\circ}$ \\
\hline 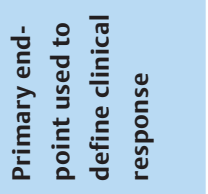 & 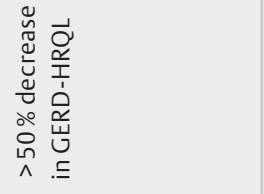 & 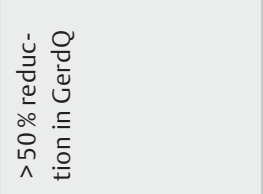 & 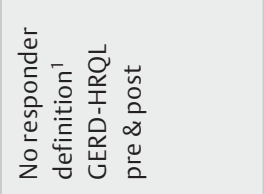 & 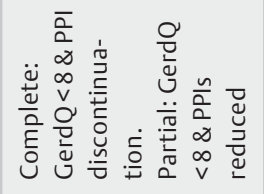 & 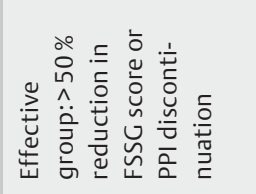 \\
\hline 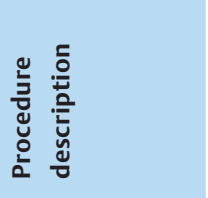 & 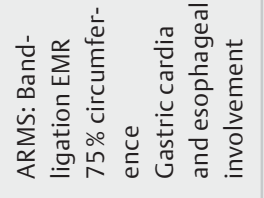 & 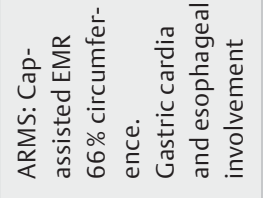 & 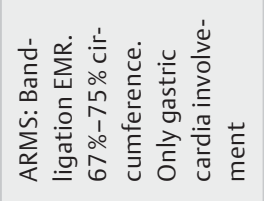 & 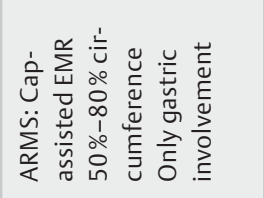 & 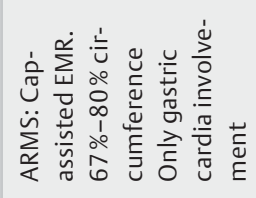 \\
\hline 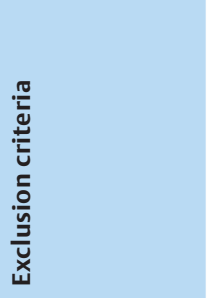 & 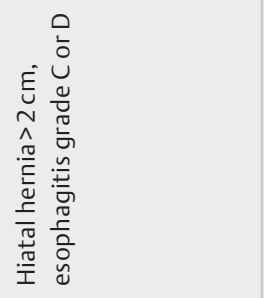 & 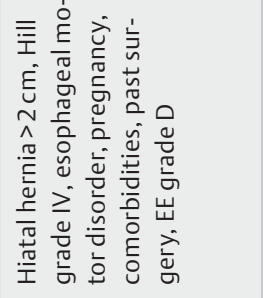 & 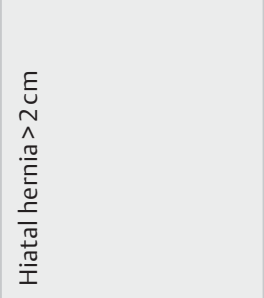 & 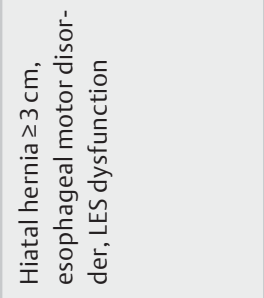 & 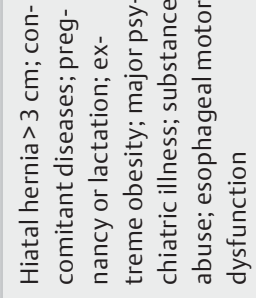 \\
\hline 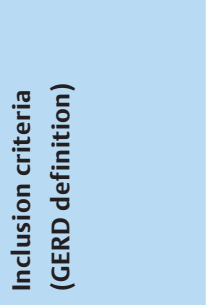 & 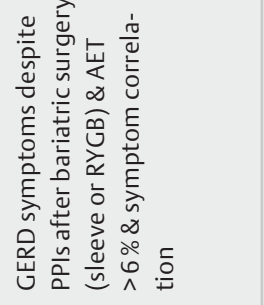 & 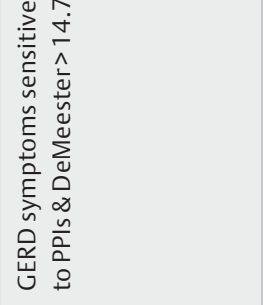 & 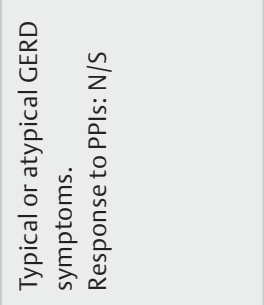 & 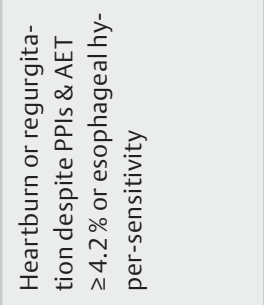 & 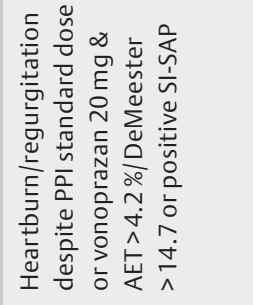 \\
\hline 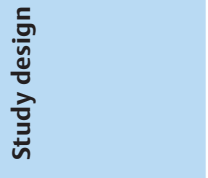 & 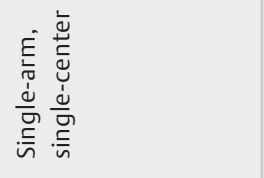 & 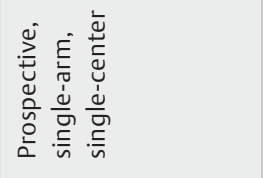 & 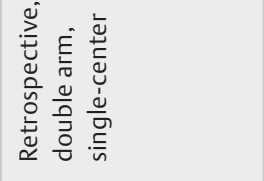 & 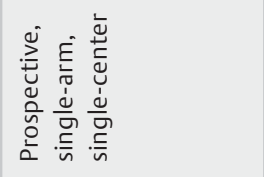 & 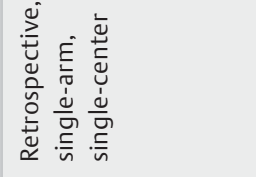 \\
\hline 莺 & 离 & 离 & 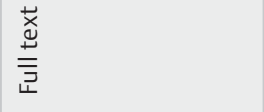 & 离 & 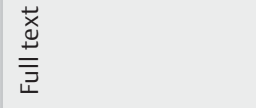 \\
\hline 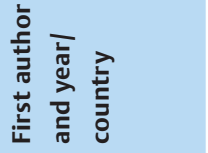 & 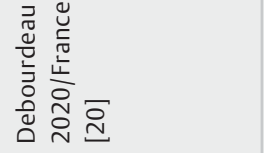 & 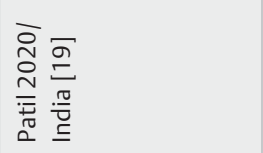 & 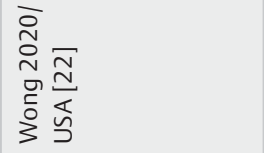 & 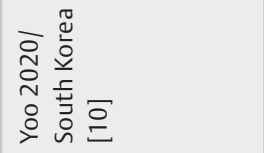 & 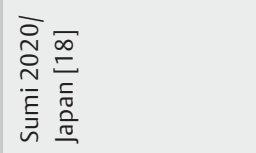 \\
\hline
\end{tabular}




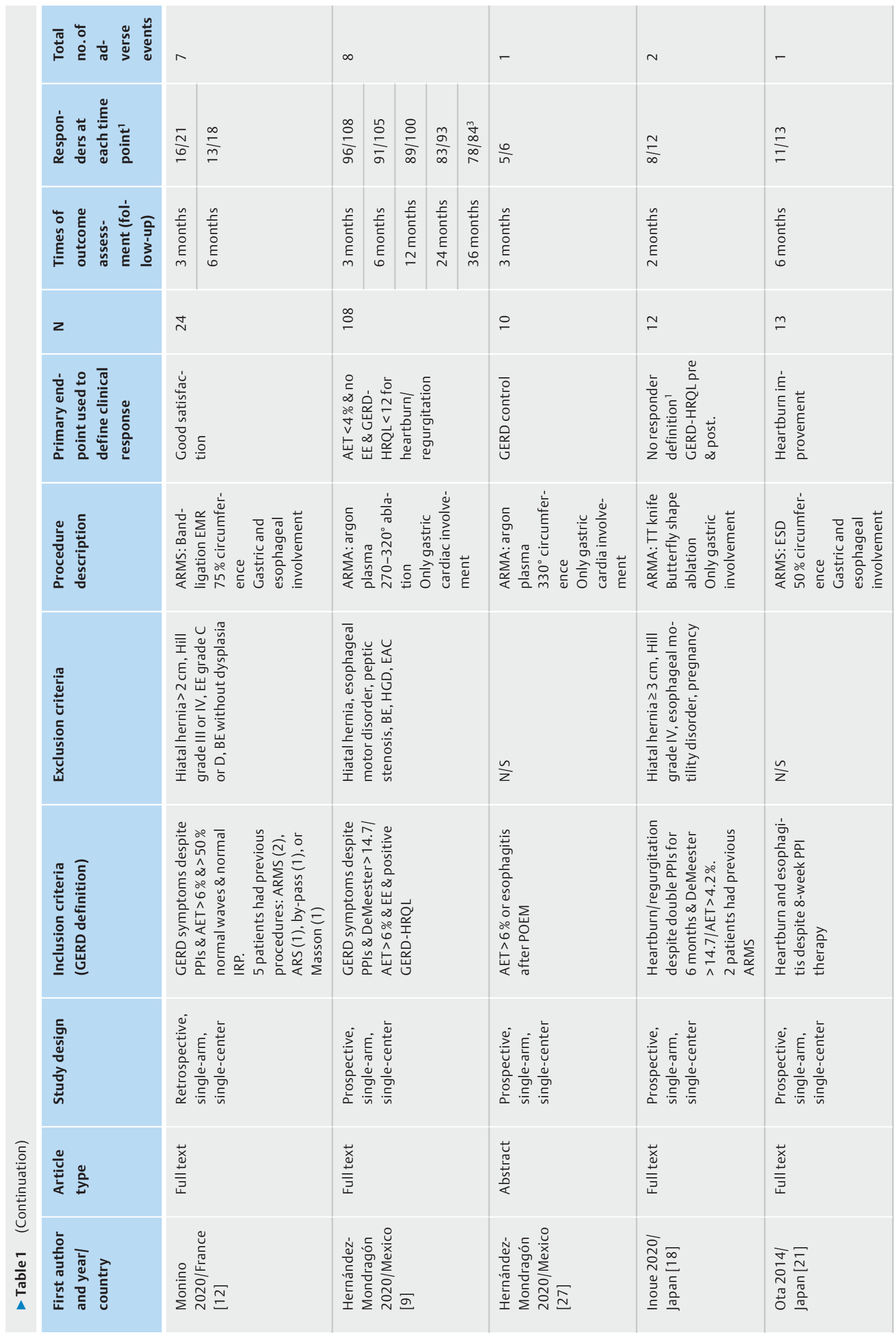




\begin{tabular}{|c|c|c|c|c|c|}
\hline 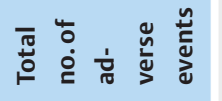 & 0 & $m$ & 0 & $m$ & 0 \\
\hline 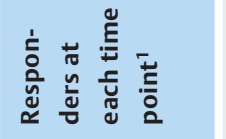 & $\frac{5}{\infty}$ & $\frac{\nabla}{\sigma}$ & $\stackrel{N}{N}$ & 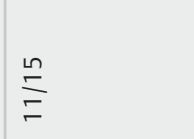 & $\frac{\varphi}{\sigma}$ \\
\hline 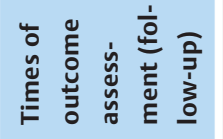 & 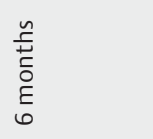 & $\begin{array}{l}\tilde{n} \\
\underline{E} \\
\underline{0} \\
\stackrel{0}{E} \\
m\end{array}$ & $\frac{n}{z}$ & 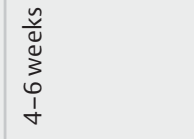 & 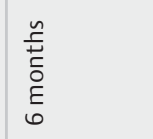 \\
\hline$z$ & $=$ & $n$ & $\simeq$ & $\stackrel{\llcorner}{\leftarrow}$ & 0 \\
\hline 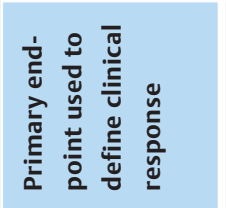 & 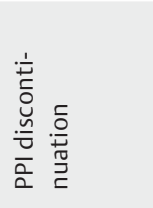 & $\frac{n}{z}$ & 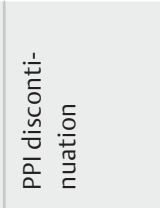 & 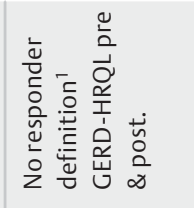 & 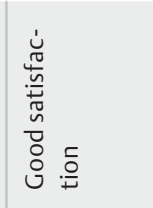 \\
\hline 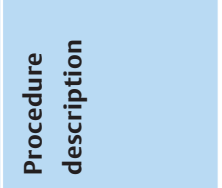 & 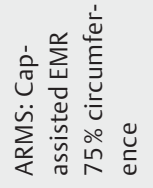 & $\sum_{\frac{\alpha}{\alpha}}^{n}$ & 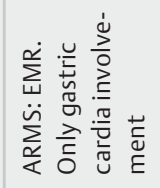 & 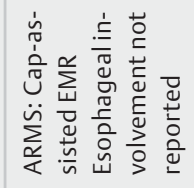 & 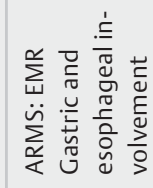 \\
\hline
\end{tabular}
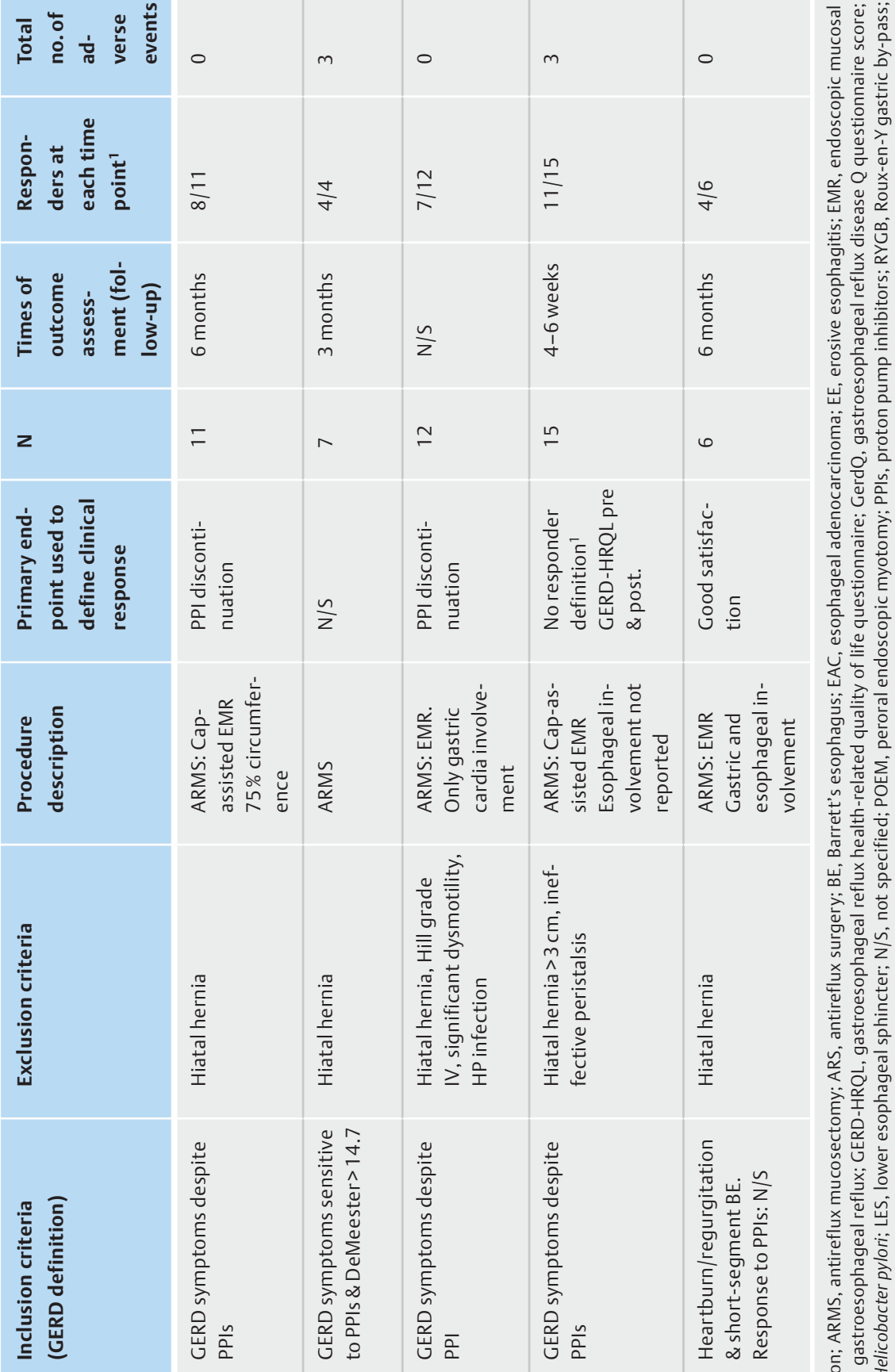

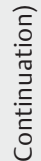
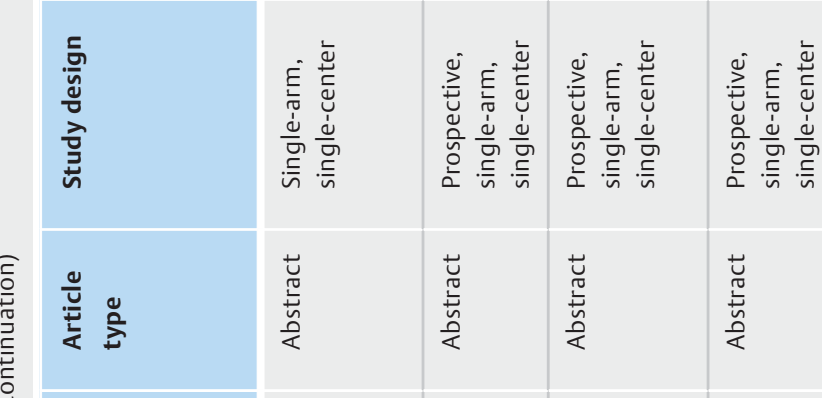

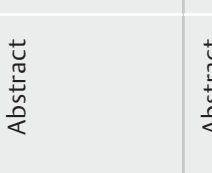




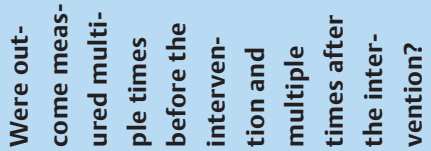

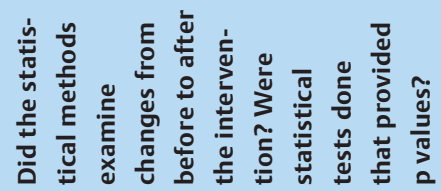

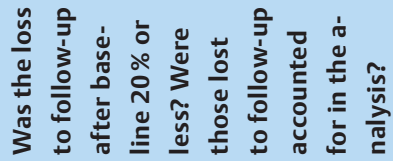

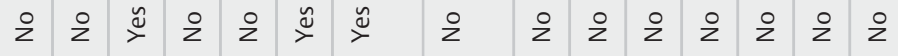

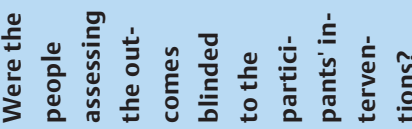

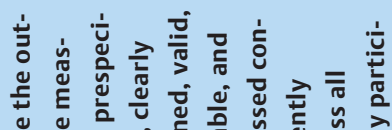

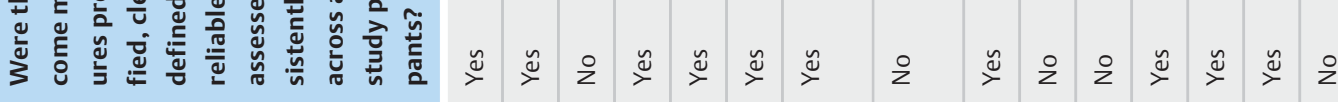

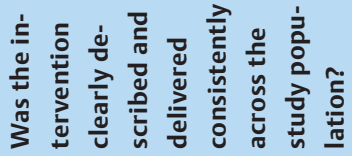

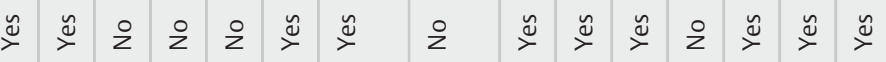

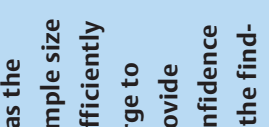

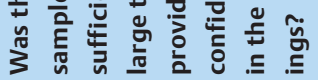

ż

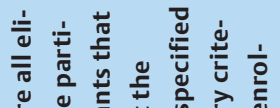

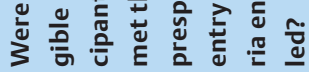

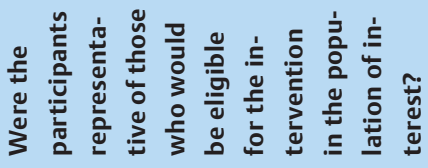

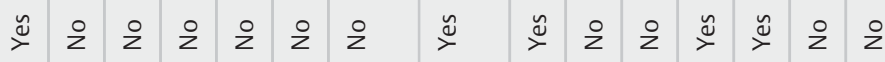

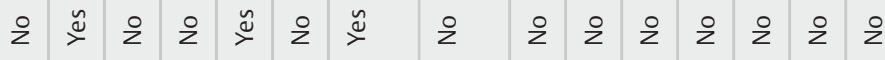

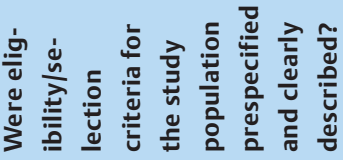


- Table 3 Clinical success and proton pump inhibitor discontinuation.

\begin{tabular}{|c|c|c|c|c|c|c|c|c|}
\hline \multirow[t]{2}{*}{ Outcome } & \multicolumn{4}{|l|}{ Short-term } & \multicolumn{4}{|l|}{1 year } \\
\hline & $\begin{array}{l}\text { No. of } \\
\text { studies; N }\end{array}$ & $\begin{array}{l}\text { Pooled propor- } \\
\text { tion/MD }\end{array}$ & $95 \% \mathrm{Cl}(\%)$ & $I^{2}(\%)$ & $\begin{array}{l}\text { No. of } \\
\text { studies; N }\end{array}$ & $\begin{array}{l}\text { Pooled propor- } \\
\text { tion/MD }\end{array}$ & $95 \% \mathrm{Cl}(\%)$ & $I^{2}(\%)$ \\
\hline Clinical success & $15 ; 430$ & $78 \%$ & $70-85$ & 54.2 & $3 ; 209$ & $72 \%$ & $47-92$ & 93 \\
\hline GERD-HRQL & $4 ; 142$ & MD 18.6, $P<0.01$ & $9.0-28.2$ & 0 & $2 ; 110$ & $\begin{array}{l}\text { MD 15.1, } \\
P<0.01\end{array}$ & $10.6-18.8$ & 0 \\
\hline GerdQ & $4 ; 189$ & MD 5.0, $P<0.01$ & $2.2-7.9$ & 0 & $1 ; 61$ & Not estimated & $\begin{array}{l}\text { Not } \\
\text { estimated }\end{array}$ & $\begin{array}{l}\text { Not } \\
\text { estimated }\end{array}$ \\
\hline $\begin{array}{l}\text { Patients } \\
\text { without PPIs }\end{array}$ & $13 ; 430$ & $63 \%$ & $51-74$ & 78.5 & $3 ; 212$ & $64 \%$ & $52-75$ & 68 \\
\hline
\end{tabular}

\section{Discussion}

Several minimally invasive endoscopic techniques have been developed in recent years to fill the existing gap between PPIs and surgery. ARMS and, more recently, ARMA, have gained increasing attention among endoscopists due to their simplicity and because they do not require costly add-on devices. Furthermore, both techniques can be performed in an ambulatory setting and do not necessarily require general anesthesia $[9,10$, 12]. This meta-analysis indicates that ARMS and ARMA are feasible and improve subjective and objective GERD-related outcomes with an acceptable safety profile.

Our study found that approximately three of four patients achieved clinical success in the short-term and that $60 \%$ could discontinue PPIs. These findings were endorsed by a significant improvement in 24-hour $\mathrm{pH}$ monitoring parameters and endoscopic esophagitis. Long-term follow-up was limited, but studies reporting outcomes at 1 and 3 years suggest that the response is durable $[9,18,19]$. These success rates mirror those reported for LARS and others endoscopic procedures $[22,28$, 29], with the advantage that both techniques can be performed at a lower cost with standard endoscopic equipment. ARMS and ARMA are thought to suppress the backflow of gastric content and enhance the $\mathrm{GO}$ J flap valve mechanism, although the underlying pathophysiological mechanism is not fully understood [18]. ARMS was incidentally devised in a patient with Barrett'srelated high-grade dysplasia who underwent ESD [7]. The resulting scar reduced the diameter of the gastric cardia opening and normalized the acid exposure time (Supplementary Fig. 3 and Supplementary Fig.4). A randomized animal study found that ARMS increased the intragastric pressure and volume required to induce fluid passage from the stomach to the esophagus. In addition, this preclinical trial found that ARMS led to a reduction in the width of the cardia [30]. Only one of the included studies in this meta-analysis provided HRM and EndoFLIP data. The integrated relaxation pressure and lower esophageal sphincter resting pressure significantly increased after ARMS, whereas GOJ distensibility decreased [10]. Hypothetically, this could reduce the number of transient lower esophageal relaxations, which play a major role in GERD pathogenesis [8, 18].

Patient selection criteria are key when selecting candidates for these procedures. Sliding hiatal hernia ( $>2$ to $3 \mathrm{~cm}$ ) was an exclusion criterion in all of the studies. It should be regarded a contraindication to the technique because the diaphragmatic crura impairment cannot be repaired endoscopically. Of the 15 studies, 10 included patients refractory to PPIs, representing $83 \%$ of the total population included in this systematic review. The definition for refractory GERD was heterogeneous, probably due to the lack of a standardized definition in the literature, but individuals with refractory GERD represent a population with a lower likelihood of a response to endoscopic and surgical therapies [3]. It should also be noted that most patients were naïve to any $\mathrm{GO}$ J endoscopic or surgical treatment, so the role of ARMS and ARMA in the presence of altered anatomy remains to be elucidated. Interestingly, one small case series reported favorable outcomes in GERD after peroral endoscopic myotomy [27]. Furthermore, ARMS was used to successfully treat patients with a history of laparoscopic sleeve gastrectomy, in whom the absence of the gastric fundus hinders LARS [20]. We believe that these selected populations and patients with good response to PPIs and chronic consumption are good candidates for the technique and merit inclusion in future studies. Thus far, the only predictor of a good response to ARMA or ARMS is a preserved flap valve at baseline (Hill grade I or II) [9].

AEs are another caveat when a new technique is developed. The rate of adverse events was lower than in recent LARS cohorts [5], which is probably the main advantage of endoscopic therapies. No study reported endoscopy-related deaths and all but two AEs could be managed conservatively. However, the risk of adverse events was slightly higher compared with Stretta and transoral incisionless fundoplication [28,31]. This was due to a higher postprocedural dysphagia rate $(7 \%)$, which is the most common ARMS- and ARMA-related AE. Nonetheless, this risk is lower than that of LARS, where nearly $23 \%$ of patients can experience long-term dysphagia [29]. According to a recent RCT, LARS-associated dysphagia has a poor response to 


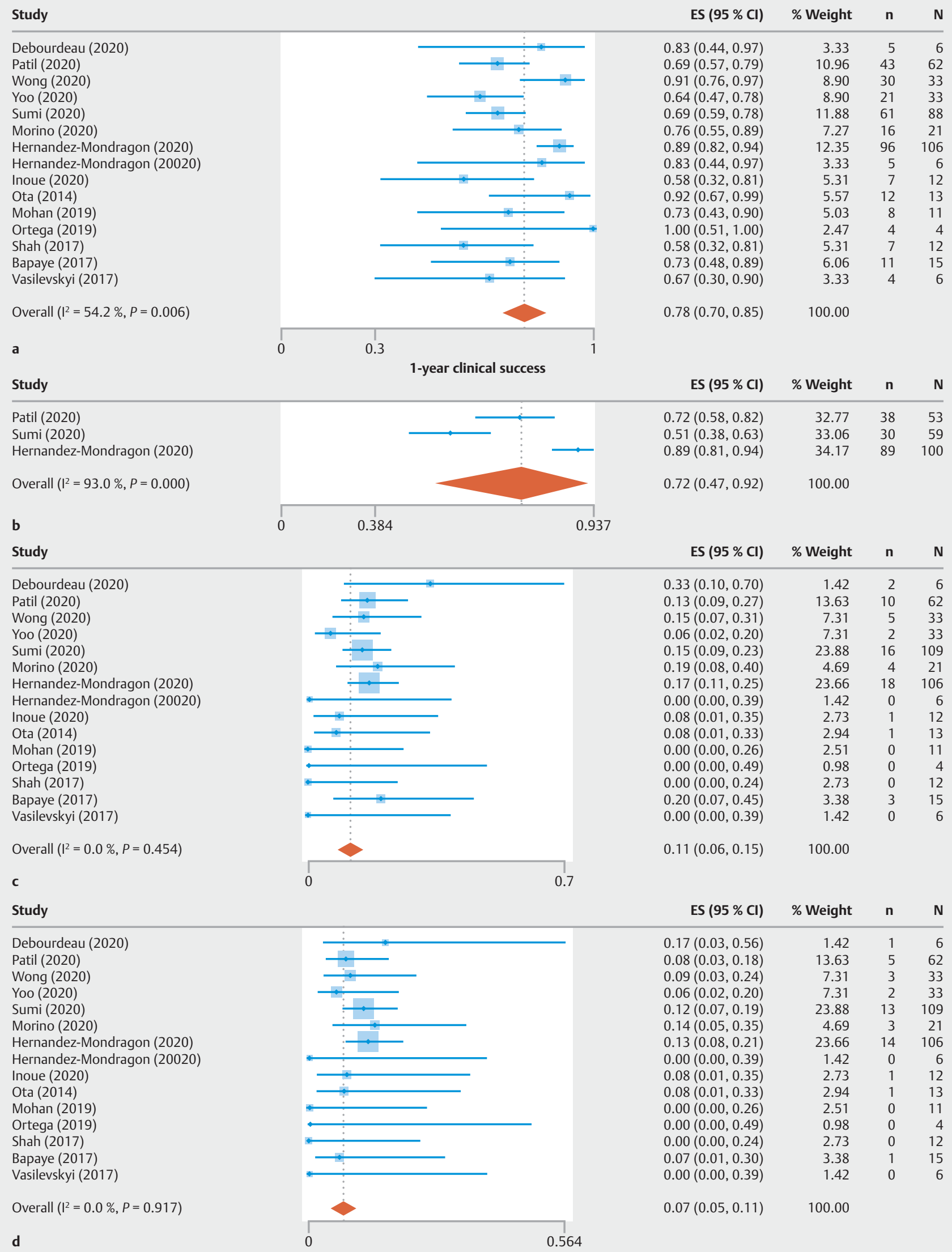

Fig. 2 Forest plots of clinical success at a short-term follow-up, $\mathbf{b}$ clinical success at 1 year, $\mathbf{c}$ adverse events, and $\mathbf{d}$ dysphagia requiring dilation. "N" denotes the total sample size and " $\mathrm{n}$ " the number of events. 
- Table4 Subgroup analysis comparing antireflux mucosectomy and antireflux mucosal ablation.

\begin{tabular}{|c|c|c|c|c|c|c|c|c|c|}
\hline \multirow[t]{2}{*}{ Outcome } & \multicolumn{4}{|c|}{ Antireflux mucosectomy } & \multicolumn{4}{|c|}{ Antireflux mucosal ablation } & \multirow[t]{2}{*}{$P$ value } \\
\hline & $\begin{array}{l}\text { No. of } \\
\text { studies; } \\
\text { N }\end{array}$ & $\begin{array}{l}\text { Pooled pro- } \\
\text { portion }\end{array}$ & $\begin{array}{l}95 \% \mathrm{Cl} \\
(\%)\end{array}$ & $I^{2}(\%)$ & $\begin{array}{l}\text { No. of } \\
\text { studies; } \\
\text { N }\end{array}$ & $\begin{array}{l}\text { Pooled } \\
\text { propor- } \\
\text { tion }\end{array}$ & $\begin{array}{l}95 \% \mathrm{Cl} \\
(\%)\end{array}$ & $I^{2}(\%)$ & \\
\hline $\begin{array}{l}\text { Short-term clinical } \\
\text { success }\end{array}$ & $12 ; 304$ & $76 \%$ & $69-82$ & 27.6 & $3 ; 126$ & $81 \%$ & $56-97$ & 0 & 0.68 \\
\hline 1-year clinical success & $2 ; 112$ & $61 \%$ & $52-70$ & 0 & $1 ; 100$ & $89 \%$ & $81-94$ & 0 & $\begin{array}{l}\text { Not esti- } \\
\text { mated }\end{array}$ \\
\hline 3-year clinical success & $1 ; 21$ & $\begin{array}{l}16 \text { out of } 21 \\
(76.2 \%)\end{array}$ & $\begin{array}{l}\text { Not esti- } \\
\text { mated }\end{array}$ & $\begin{array}{l}\text { Not esti- } \\
\text { mated }\end{array}$ & $1 ; 108$ & $\begin{array}{l}78 \text { of } 108 \\
(72.2 \%)\end{array}$ & $\begin{array}{l}\text { Not esti- } \\
\text { mated }\end{array}$ & $\begin{array}{l}\text { Not esti- } \\
\text { mated }\end{array}$ & $\begin{array}{l}\text { Not esti- } \\
\text { mated }\end{array}$ \\
\hline $\begin{array}{l}\text { Patients without PPIs at } \\
\text { short-term follow-up }\end{array}$ & $11 ; 310$ & $62 \%$ & $48-76$ & 79.8 & $2 ; 120$ & $70 \%$ & $61-78$ & 0 & 0.33 \\
\hline $\begin{array}{l}\text { Patients without PPIs at } \\
1 \text { year }\end{array}$ & $2 ; 109$ & $61 \%$ & $52-70$ & 0 & $1 ; 100$ & $68 \%$ & $58-76$ & 0 & 0.28 \\
\hline Overall adverse events & $12 ; 325$ & $10 \%$ & $6-15$ & 9.4 & $3 ; 126$ & $13 \%$ & $7-20$ & 0 & 0.56 \\
\hline $\begin{array}{l}\text { Dysphagia requiring } \\
\text { dilation }\end{array}$ & $12 ; 325$ & $7 \%$ & $4-10$ & 0 & $3 ; 126$ & $10 \%$ & $4-16$ & 0 & 0.39 \\
\hline
\end{tabular}

endoscopic dilation and often requires reintervention [32], whereas post-ARMS/ARMA dysphagia can be successfully controlled by small-caliber balloon dilation $[8,9,11,12,18-20]$. Besides, fundoplication can alter gastric emptying through inadvertent vagal nerve injury, causing dyspeptic symptoms. This adverse effect is not expected with ARMS or ARMA because the mucosal damage is not deep enough to affect relevant neurological structures. Notably, significant fibrosis around the GOJ is not common and our data indicate that LARS is not hampered by ARMS or ARMA [9, 12].

Rescue ARMS and ARMA seem feasible and can provide symptom control in some patients, although data were insufficient to elucidate which patients will benefit from a second procedure. The fact that redo ARMA $(n=22)$ was more common than redo ARMS $(n=4)$ could possibly be indicative that repeat ARMA is technically easier. The scar induced by the first procedure often precludes adequate submucosal injection that hampers mucosectomy but not ablation.

Several technical refinements have been proposed to improve feasibility and safety. First, because ARMS can be successfully performed by either cap- or band-ligation EMR, the use of ESD in this setting should be abandoned. Second, our subgroup analysis suggested that resection of the esophageal mucosa does not improve the rate of clinical success, which is why our group and others advocate a gastric cardia-only technique in search of simplicity. Third, a "butterfly"-shaped resection or ablation, sparing $1 \mathrm{~cm}$ of normal mucosa along the greater and lesser curvature, could reduce the risk of stenosis, although no robust data are yet available [18]. Fourth, adequate submucosal injection is critical to minimize the risk of muscularis propria injury. In our systematic review, all perforations occurred in the ARMS group. This poses the question of whether ARMA should replace ARMS, given that ablation achieved similar GERD control. However, the body of evidence is less for ARMA and no head-to-head studies were found; therefore, we believe that this debate remains open and requires further research. Fifth, our initial ARMA study used a Triangle Tip Knife (Olympus) for mucosal ablation [8], but APC seems equally effective and could reduce procedure-related costs $[9,27]$.

Our study has limitations. It could be argued that the definition of clinical success was not uniform, which could be explained by the lack of expert consensus on how to measure success in GERD therapies. To overcome this limitation, we also pooled data from validated clinical questionnaires, PPI consumption, endoscopic esophagitis, and 24-hour pH monitoring and conducted a sensitivity analysis using a commonly used definition in recent studies. All analyses supported the efficacy of ARMS and ARMA. Another limitation is that all studies were single-arm and no RCTs were found. The placebo effect cannot be ruled out, although it seems unlikely that this would account for ARMA/ARMS efficacy, considering that the response rate to placebo in GERD clinical trials is around 20\% [33]. Nonetheless, we agree with guidelines from the European Society of Gastrointestinal Endoscopy recommending that these techniques be performed within research protocols due to the lack of longterm and controlled data [6].

\section{Conclusions}

In conclusion, this meta-analysis comprising 15 nonrandomized studies shows that ARMA and ARMS seem feasible, safe, and effective for patients with GERD. Our findings indicate that these techniques deserve further testing in RCTs and provide valuable information for their design. 


\section{Competing interests}

Dr. Inoue is an advisor for and received educational grants from Olympus Medical Systems Corporation. He has also received educational grants from the Takeda Pharmaceutical Company and is an advisor for the Top Corporation.

\section{References}

[1] Eusebi LH, Ratnakumaran R, Yuan Y et al. Global prevalence of, and risk factors for, gastro-oesophageal reflux symptoms: a meta-analysis. Gut 2018; 67: 430-440

[2] GBD 2017 Gastro-oesophageal Reflux Disease Collaborators. The global, regional, and national burden of gastro-oesophageal reflux disease in 195 countries and territories, 1990-2017: a systematic analysis for the Global Burden of Disease Study 2017. Lancet Gastroenterol Hepatol 2020; 5: 561-581

[3] Yadlapati R, Vaezi MF, Vela MF et al. Management options for patients with GERD and persistent symptoms on proton pump inhibitors: recommendations from an expert panel. Am J Gastroenterol 2018; 113 : 980-986

[4] de la Coba Ortiz C, Argüelles Arias F, Martín de Argila de Prados C et al. Proton-pump inhibitors adverse effects: a review of the evidence and position statement by the Sociedad Española de Patología Digestiva. Rev Esp Enferm Dig 2016; 108: 207-224

[5] Singhal S, Kirkpatrick DR, Masuda T et al. Primary and redo antireflux surgery: outcomes and lessons learned. J Gastrointest Surg 2018; 22: 177-186

[6] Weusten BLAM, Barret M, Bredenoord AJ et al. Endoscopic management of gastrointestinal motility disorders - part 2: European Society of Gastrointestinal Endoscopy (ESGE) Guideline. Endoscopy 2020; 52: 600-614

[7] Inoue $\mathrm{H}$, Ito $\mathrm{H}$, Ikeda $\mathrm{H}$ et al. Anti-reflux mucosectomy for gastroesophageal reflux disease in the absence of hiatus hernia: A pilot study. Ann Gastroenterol 2014; 27: 346-351

[8] Inoue $\mathrm{H}$, Tanabe M, de Santiago ER et al. Anti-reflux mucosal ablation (ARMA) as a new treatment for gastroesophageal reflux refractory to proton pump inhibitors: a pilot study. Endosc Int Open 2020; 8: E133E138

[9] Hernández Mondragón OV, Zamarripa Mottú RA, García Contreras LF et al. Clinical feasibility of a new antireflux ablation therapy on gastroesophageal reflux disease (with video). Gastrointest Endosc 2020; 92: $1190-1201$

[10] Yoo I, Ko WJ, Kim HS et al. Anti-reflux mucosectomy using a cap-assisted endoscopic mucosal resection method for refractory gastroesophageal disease: a prospective feasibility study. Surg Endosc 2020; 34: 1124-1131

[11] Bapaye A, Mahadik M, Pujari R et al. Anti-reflux mucosectomy (ARMS) for refractory GERD-Initial clinical experience. J Gastroenterol Hepatol 2017; 32: 255

[12] Monino L, Gonzalez JM, Vitton V et al. Antireflux mucosectomy band in treatment of refractory gastroesophageal reflux disease: A pilot study for safety, feasibility and symptom control. Endosc Int Open 2020; 8: E147-E154 doi:10.1055/a-1038-4012

[13] Ma L-L, Wang Y-Y, Yang Z-H et al. Methodological quality (risk of bias) assessment tools for primary and secondary medical studies: what are they and which is better? Mil Med Res 2020; 7: 7

[14] Higgins JP, Thomas J, Chandler J et al. Cochrane handbook for systematic reviews of interventions. John Wiley \& Sons; 2019
[15] Barendregt J], Doi SA, Lee YY et al. Meta-analysis of prevalence. J Epidemiol Community Health 2013; 67: 974-978

[16] Wan X, Wang W, Liu J et al. Estimating the sample mean and standard deviation from the sample size, median, range and/or interquartile range. BMC Med Res Methodol 2014; 14: 135

[17] Spechler SJ, Hunter JG, Jones KM et al. Randomized Trial of Medical versus Surgical Treatment for Refractory Heartburn. N Engl J Med 2019; 381: 1513-1523

[18] Sumi K, Inoue H, Kobayashi Y et al. Endoscopic treatment of proton pump inhibitor-refractory gastroesophageal reflux disease with antireflux mucosectomy: Experience of 109 cases. Dig Endosc 2020: doi: $10.1111 /$ den. 13727

[19] Patil G, Dalal A, Maydeo A. Feasibility and outcomes of anti-reflux mucosectomy for proton pump inhibitor dependent gastroesophageal reflux disease: First Indian study (with video). Dig Endosc 2020; 32: 745-752

[20] Debourdeau A, Vitton V, Monino L et al. Antireflux Mucosectomy Band (ARM-b) in treatment of refractory gastroesophageal reflux disease after bariatric surgery. Obes Surg 2020; 30: 4654-4658

[21] Ota K, Takeuchi T, Harada S et al. A novel endoscopic submucosal dissection technique for proton pump inhibitor-refractory gastroesophageal reflux disease. Scand J Gastroenterol 2014; 49: 14091413

[22] Wong H], Su B, Attaar M et al. Anti-reflux mucosectomy (ARMS) results in improved recovery and similar reflux quality of life outcomes compared to laparoscopic Nissen fundoplication. Surg Endosc 2020: doi:10.1007/s00464-020-08144-9

[23] Vasilevskiy DI, Bagnenko SF, Smirnov A et al. Antireflux mucosectomy (Arms) in the treatment of patients with gerd and columnar-cell lined (barrett's) esophagus. First experiences. Surg Endosc 2017; 31: S405

[24] Shah R, Maydeo AP, Dhir V. Anti reflux mucosectomy (ARMS) for refractory gastro esophageal reflux disease (GERD)-are we there yet? United European Gastroenterol J 2017; 5: A354-A355

[25] Ortega A, Rosón P, Fern F et al. Antireflux mucosectomy. preliminary results of a prospective study. Endoscopy 2019; 51: S240-S241

[26] Mohan PrasadVG, Appadurai S, Suman MP. Anti-reflux mucosectomy (ARMS) for refractory GERD-Our 3yrs experience. J Gastroenterol Hepatol 2019; 34: 232

[27] Hernández Mondragón OV, Pintor JC, Gutierrez Aguilar RA et al. Antireflux ablation therapy (ARAT), for reflux disease after poem procedure. Early clinical experience. Gastrointest Endosc 2020; 91: AB130

[28] Fass R, Cahn F, Scotti D] et al. Systematic review and meta-analysis of controlled and prospective cohort efficacy studies of endoscopic radiofrequency for treatment of gastroesophageal reflux disease. Surg Endosc 2017; 31: 4865-4882

[29] Garg SK, Gurusamy KS. Laparoscopic fundoplication surgery versus medical management for gastro-oesophageal reflux disease (GORD) in adults. Cochrane Database Syst Rev; 2015: CD003243

[30] Li X, Zhang W, Chen M et al. A Prospective randomized trial to assess the antireflux effect of antireflux mucosectomy in the porcine model. Gastroenterol Res Pract 2019: doi:10.1155/2019/3286738

[31] McCarty TR, Itidiare M, Njei B et al. Efficacy of transoral incisionless fundoplication for refractory gastroesophageal reflux disease: a systematic review and meta-analysis. Endoscopy 2018; 50: 708-725

[32] Schuitenmaker JM, van Hoeij FB, Schijven MP et al. Pneumatic dilation for persistent dysphagia after antireflux surgery, a multicentre singleblind randomised sham-controlled clinical trial. Gut 2021: doi:10.1136/gutjnl-2020-322355

[33] Cremonini F, Ziogas DC, Chang HY et al. Meta-analysis: the effects of placebo treatment on gastro-oesophageal reflux disease. Aliment Pharmacol Ther 2010; 32: 29-42 